\title{
Soluble ST2 as a Diagnostic and Prognostic Marker for Acute Heart Failure Syndromes
}

\author{
Queen Henry-Okafor ${ }^{1}$, Sean P. Collins ${ }^{1}$, Cathy A. Jenkins ${ }^{1}$, Karen F. Miller ${ }^{1}$, David J. Maron ${ }^{1}$, \\ Allen J. Naftilan ${ }^{1}$, Neal Weintraub ${ }^{4}$, Gregory J. Fermann ${ }^{2}$, John McPherson ${ }^{1}$, Santosh Menon ${ }^{3}$, \\ Douglas B. Sawyer ${ }^{1}$ and Alan B. Storrow ${ }^{1, *}$
}

${ }^{1}$ Department of Cardiovascular Medicine (QHO, DJM, AJN, JM, DBS), the Department of Emergency Medicine (QHO,
KFM, DJM, ABS), and the Department of Biostatistics (CAJ), Vanderbilt University School of Medicine, Nashville, TN,
USA
${ }^{2}$ Department of Emergency Medicine (SPC, NW, GJF), University of Cincinnati, OH, USA
${ }^{3}$ Department of Cardiovascular Medicine (SM), The Ohio Heart \& Vascular Center, The Christ Hospital in Cincinnati,
OH, USA
${ }^{4}$ Division of Cardiology, Department of Internal Medicine, University of Cincinnati, OH, USA

Abstract: Objectives: We investigated the association of sST2 with diagnostic and prognostic outcomes and assessed whether it aids B-natriuretic peptide (BNP) in diagnosing and predicting outcomes in emergency department (ED) patients with suspected AHFS.

\begin{abstract}
Methods: We recruited patients who presented to the ED of 3 tertiary hospitals with signs or symptoms of AHFS and met modified Framingham criteria for AHFS. Outcome measures were a final diagnosis of AHFS and 5-and 30-day adverse events.

Results: In the 295 subjects with sST2 available, the median sST2 was $0.20 \mathrm{ng} / \mathrm{ml}$ (IQR=0.10, 0.34). Although unadjusted analyses indicated sST2 was significantly associated with the diagnosis of AHFS ( $\mathrm{p}=0.02)$, this was not so in the adjusted analysis $(\mathrm{p}=0.33)$. Moderately low diagnostic utility was noted with an AUC of $0.62(95 \% \mathrm{CI}=0.56,0.69)$. Similar sST2 test characteristics were seen when BNP was restricted between 100 and $500 \mathrm{pg} / \mathrm{ml}$. While sST2 was associated with AHFS readmission at 30-days ( $\mathrm{p}=0.04)$, in the adjusted analyses it was not associated with adverse events.

Conclusion: In patients with signs or symptoms of AHFS, unadjusted analyses indicated that sST2 was significantly associated with the diagnosis of AHFS and with 30-day AHFS recidivism. However, the associations did not carry over to adjusted analyses, and sST2 did not add significant information with regard to explaining the diagnostic and prognostic variability of BNP.
\end{abstract}

Keywords: Soluble ST2, Acute heart failure, Diagnosis, Prognosis.

\section{INTRODUCTION}

Acute heart failure syndromes (AHFS) are a major public health concern worldwide [1]. The majority of patients with signs or symptoms of AHFS receive initial evaluation and treatment in the emergency department (ED) setting [2]. Therefore, there is a growing need to improve the ability of the emergency physician to effectively and safely manage low-risk patients and hence avoid unnecessary hospitalizations. To this end, novel biomarkers are being examined to determine their role in AHFS diagnosis and risk prediction $[3,4]$.

*Address correspondence to this author at the Vice Chairman for Research and Academic Affairs Department of Emergency Medicine Vanderbilt University $131321^{\text {st }}$ Avenue, South 703 Oxford House Nashville, TN, 37232-4700, USA; Tel: (615) 936-5934; Fax: (615) 936-4490;

E-mail: alan.b.storrow@vanderbilt.edu
ST2, a member of the interleukin (IL)-1 receptor family, is rapidly emerging as a novel biomarker for cardiac strain $[5,6]$. The ST2 gene is induced by the stimulation of various proliferation-inducing agents including platelet-derived growth factor (PDGF), fibroblast growth factor (FGF), and lysophosphatidic acid [7-9]. It has been shown that the IL33/ST2 system plays a part in the progression of atherosclerotic vascular disease. High plasma concentrations of sST2 are reportedly common in AHFS and have been suggested to indicate increased risk of mortality [10].

The emergency diagnosis of AHFS begins with clinical presentation, radiographic examination and, when there is diagnostic indecision, a natriuretic peptide level $[11,12]$. Btype Natriuretic Peptide (BNP) has been accepted in the scientific community as useful for the diagnosis and risk stratification of AHFS [13, 14]. At BNP levels less than 100 $\mathrm{pg} / \mathrm{ml}$ AHFS is uncommon [15]. Unfortunately, the diagnos- 
tic accuracy of BNP levels between 100 to $500 \mathrm{pg} / \mathrm{ml}$ has been limited; hence, this is often referred to as the "BNP gray zone" [16]. It is unknown if the addition of sST2 to BNP levels in the gray zone will add increased utility. Among patients presenting to the ED with signs or symptoms of AHFS, our central hypotheses are that SST2 concentrations: 1) are independently associated with a diagnosis of AHFS; 2) add diagnostic utility to gray zone concentrations of BNP; and 3) are independently associated with AHFS prognosis.

\section{METHODS}

\section{Study Population and Data Collection}

We utilized a cohort of patients from the STRATIFY study, an ongoing prospective cohort study of adults presenting with signs and symptoms of possible AHFS to three different tertiary care EDs. To be eligible for inclusion in STRATIFY, patients had to meet the modified Framingham criteria for AHFS (Table 1) and had to be enrolled within three hours of first ED physician contact. This study was approved by the Institutional Review Boards of the recruiting centers.

Trained study coordinators approached candidates who had qualified based on the above criteria. The study coordinators explained the study purpose and protocol to potential candidates. Written informed consent was obtained from patients who indicated their willingness to participate. Detailed demographic and clinical data were collected using questionnaires administered to the patients with verification using medical records. Further, additional samples of serum, plasma and whole blood were processed and stored at the time of enrollment for future analyses. Study coordinators performed follow-up interviews at both 5 and 30-days to document adverse events with verification via medical records. When difference occurred, medical record information took precedence over interview responses. If the patient was still in the hospital at 5-day or 30-day follow-up, then an inpatient visit was made by the study coordinators. For the current substudy, we evaluated a consecutive sample of patients from STRATIFY who had plasma samples available for analysis.

\section{sST2 Assay}

Soluble human ST2 protein was measured from frozen, previously unthawed plasma samples that were obtained at enrollment and stored at $-80^{\circ} \mathrm{C}$ until time of assay. Analysis of the concentrations of sST2 was done with an enzyme linked immunosorbent assay (Medical \& Biological Laboratories Co., Woburn, Massachusetts). The assay uses two monoclonal antibodies against two different epitopes of human ST2. Samples to be measured or standards are incubated in the microwells coated with anti-human ST2 monoclonal antibody. After washing, peroxidase-conjugated anti-human ST2 monoclonal antibody is added into the microwells and incubated. After another washing, the peroxidase substrate premixed with chromogen is added and allowed to incubate for an additional period of time. An acid solution is then added to each microwell to terminate the enzyme reaction and to stabilize the developed color. The optical density (O.D.) of each microwell is then measured at $450 \mathrm{~nm}$ using a microplate reader.

\section{B-type Natriuretic Peptide Assay}

Blood samples for measurement of BNP plasma levels were collected at enrollment by venipuncture into ethylene diamine tetra acetic acid (EDTA) tubes. The blood samples were centrifuged and plasma was removed, aliquoted and frozen at $-80^{\circ} \mathrm{C}$ before analysis. The whole blood was then analyzed in triplicate with the Triage BNP assay (Biosite Diagnostics Inc., San Diego, California). The Triage Meter is used to measure the BNP concentration by detecting a fluorescent signal that reflects the amount of BNP in the sample. Testing for BNP levels was performed by the Vanderbilt University Laboratory Services. BNP levels $\geq 100 \mathrm{pg} / \mathrm{ml}$ were considered to be abnormal.

\section{Criterion Standard}

Outcomes of interest were diagnosis of AHFS as well as 5-and 30-day outcomes. We utilized an expert panel of three cardiologists on the academic faculty at Vanderbilt University who were blinded to sST2 results to determine if AHFS was primary, present but not primary, or not present in each of the patients. The diagnosis was dichotomized for the

Table 1. Modified Framingham Criteria for Congestive Heart Failure (CHF)

\begin{tabular}{|l|l|}
\hline \multicolumn{1}{|c|}{ Major Criteria } & \multicolumn{1}{|c|}{ Minor Criteria } \\
\hline \hline Paroxysmal nocturnal dyspnea & Extremity edema \\
\hline Neck vein distention & Nocturnal cough \\
\hline Rales & Dyspnea on ordinary exertion \\
\hline Radiographic cardiomegaly (increasing heart size on chest radiography) & Hepatomegaly \\
\hline Acute pulmonary edema & Pleural effusion \\
\hline History of CHF & Tachycardia (heart rate $>120$ beats/min.) \\
\hline Hepatojugular reflux & \\
\hline Positive screen for signs and symptoms of CHF requires the simultaneous presence of at least 2 major criteria or 1 major criterion in conjunction with 2 minor criteria.
\end{tabular}


analysis into: 1) AHFS present (primary, present but not primary); or 2) AHFS not present. Patients' medical records and other test results were available to our expert panel. If two cardiologists agreed on the diagnosis, a consensus was reached. If all three cardiologists disagreed on the diagnosis, a fourth cardiologist adjudicated the decision.

Five and 30-day adverse events included: 1) prolonged hospitalization ( $\geq 5$ days following ED presentation); 2) repeat ED visit; 3) rehospitalization; 4) major cardiac events, or 5) mortality. We defined major cardiac events as: acute coronary syndrome (ACS), cardiac resynchronization therapy (CRT), insertion of implantable cardioverter defibrillator (ICD), emergent renal dialysis, endotracheal intubation, cardiac transplantation, coronary artery bypass graft, mechanical cardiac support, percutaneous coronary intervention, palliative care/hospice, sudden death with defibrillation or cardiopulmonary resuscitation.

\section{Statistical Analyses}

The statistical programming language $\mathrm{R}$, version 2.11.1 [17-20] and Stata Version 11.1 [21] were used to perform statistical analyses. Descriptive statistics on demographic data are presented as median (interquartile range, IQR) or frequency $(\%)$, as appropriate.

\section{Hypothesis 1: sST2's Diagnostic Utility in Determining the} Presence of AHFS.

The diagnostic utility of sST2 was assessed using a ROC curve. Its associated AUC was calculated as a measure of sST2 diagnostic utility in indicating the presence of AHFS.
The association of the diagnostic outcome with sST2 was assessed using univariate (Wilcoxon Rank Sum test) and multivariable logistic regression. In the multivariable analysis a priori selected confounders were selected including history of aspirin and steroid use, age, gender, race, and body mass index (BMI).

\section{Hypothesis 2: sST2 Utilization with BNP in Determining the Presence of AHFS.}

The diagnostic utility of sST2 in conjunction with BNP (overall and restricted to subjects with BNP values between 100 and $500 \mathrm{pg} / \mathrm{ml}$ ) was assessed by testing the equality of AUCs for the full and reduced models using a chi-square test [22]. The 'full model' regressed the diagnostic outcome on $\mathrm{BNP}, \mathrm{sST} 2$, prior aspirin and steroid use, age, gender, race, and BMI. The 'reduced model' omitted sST2 from the 'full model'. Likelihood (LR) tests comparing the 'full' and 'reduced models' were used to assess whether sST2 added significant information to the model. If the LR test indicated that SST2 helped BNP in explaining the variability in the outcome, the model was refit treating $\mathrm{sST} 2$ as a potential effect modifier.

\section{Hypothesis 3: The Association of sST2 with Adverse Out- comes.}

The association between sST2 and 5-and 30-day outcomes was addressed using both unadjusted and adjusted analyses. The Wilcoxon Rank Sum test was used to test the unadjusted association of sST2 with outcomes. Multivariable logistic regression was used to assess the association of sST2 with outcomes after adjusting for prior aspirin and steroid use, age, gender, race, and obesity defined as BMI [Ibs/in $\left.{ }^{2}\right]$.

Table 2. Demographics and Baseline Variables

\begin{tabular}{|c|c|c|}
\hline Variables & n (\%) & M (IQR) \\
\hline Age & & $63(52,75)$ \\
\hline \multicolumn{3}{|l|}{$\overline{\operatorname{Sex}}$} \\
\hline Male & $168(57)$ & \\
\hline \multicolumn{3}{|l|}{ Race } \\
\hline Other & $1(0)$ & \\
\hline \multicolumn{3}{|l|}{ Outpatient aspirin use } \\
\hline No & $152(52)$ & \\
\hline Yes & $143(48)$ & \\
\hline No HF & $132(45)$ & \\
\hline $\mathrm{HF}$ & $163(55)$ & \\
\hline sST2 & & $0.20(0.10,0.34)$ \\
\hline BMI & & $30(25,38)$ \\
\hline $\mathrm{BNP} \dagger$ & & $350(98,926)$ \\
\hline
\end{tabular}

Total $\mathrm{n}=295 ; \nmid \mathrm{n}=251$. M, Median; IQR, Interquartile range; AHFS, Acute Heart Failure Syndromes; COG, Cardiology Oversight Group; HF, Heart Failure; BMI, body mass index; $\mathrm{BNP}, \mathrm{B}-$ natriuretic peptide. 


\section{RESULTS}

\section{Study Population Description}

Soluble ST2 data were collected on 375 subjects. Of those, 1 subject withdrew consent; 42 had missing cardiology panel values; 9 were lost to follow-up at either the 5 or 30-day mark; 27 had missing BNP values; and 1 had incomplete 5-day follow-up information. In the end, 295 had complete data available for this analysis. Twenty-eight subjects had out-of-range sST2 values that were assigned to half the value of the detectable limit, $0.026 \mathrm{ng} / \mathrm{ml}$. The median age was $63(\mathrm{IQR}=52,75), 168(57 \%)$ were male, $213(72 \%)$ were Caucasian, and 81 (27\%) were African American (Table 2). Median sST2 was $0.20 \mathrm{ng} / \mathrm{ml}(\mathrm{IQR}=0.09,0.34)$ and median BNP was $350 \mathrm{pg} / \mathrm{ml}(\mathrm{IQR}=98,926)$. A total of $163(55 \%)$ subjects were judged to have AHFS by our expert panel.

\section{Association of sST2 with Diagnostic Outcome}

sST2 concentrations were higher in subjects with AHFS than in those without AHFS. Median sST2 in subjects with AHFS was $0.23 \mathrm{ng} / \mathrm{ml}(\mathrm{IQR}=0.11,0.41)$, versus $0.17 \mathrm{ng} / \mathrm{ml}$ $(\mathrm{IQR}=0.08,0.29)$ in those without AHFS. Unadjusted analyses indicated sST2 was significantly associated with a diagnosis of AHFS $(\mathrm{p}=0.02)$. However, this finding did not hold up in the adjusted analysis $(\mathrm{OR}=1.21 ; 95 \% \mathrm{CI}=0.83,1.76$; $\mathrm{p}=0.33)$. The AUC for $\mathrm{sST} 2$ was $0.62(95 \% \mathrm{CI}=0.56,0.69)$ suggesting moderately low diagnostic utility after adjusting for history of aspirin and steroid use, age, gender, race, and BMI (Fig. 1).

\section{Utility of sST2 with BNP}

Likelihood ratio (LR) tests failed to show that sST2 aided BNP in explaining the variability in both diagnosis and prognosis after adjusting for outpatient use of aspirin and steroids, age, gender, race, and BMI (Table 5). The AUCs for the full and reduced models were both $0.75(95 \%$ $\mathrm{CI}=0.70,0.81 ; \mathrm{p}=1.0)$. Similar results were noted for the prognostic outcome when 30-day recidivism was for allcause (LR test statistic for main model $=0.03 ; \mathrm{p}=0.87$ ) as well as for 30-day AHFS recidivism (LR test statistic for main model $=0.18 ; p=0.68$ ). Five-day all-cause or AHFS recidivism showed similar findings.

\section{Utility of sST2 with BNP levels of $100-500 \mathrm{pg} / \mathrm{ml}$}

Ninety-nine subjects had borderline elevation of BNP levels $(100-500 \mathrm{pg} / \mathrm{ml})$. Their median BNP level was 280 $\mathrm{pg} / \mathrm{ml}(\mathrm{IQR}=174,352)$ and median sST2 level was 0.14 $\mathrm{ng} / \mathrm{ml}(\mathrm{IQR}=0.09,0.25)$. Our panel of cardiologists determined that $50(51 \%)$ of these subjects had AHFS while 49 $(49 \%)$ did not. We repeated the analysis done for BNP overall on the subset of patients with BNP values in the gray zone of $100-500 \mathrm{pg} / \mathrm{ml}$. The results were similar to the previous analysis which showed $\mathrm{sST} 2$ did not add significant information to BNP in explaining the variability in the diagnosis or prognosis (Table 5). Similarly, the AUCs for the full and reduced models with the diagnostic outcome were 0.76 $(95 \% \mathrm{CI}=0.67,0.86)$ and $0.75(95 \% \mathrm{CI}=0.66,0.85)$ indicating that sST2 failed to show improvement in the diagnostic utility of BNP in the indeterminate zone $(p=0.62)$. Results from LR tests assessing whether sST2 added value to BNP levels in the gray zone were similar for subjects with 30-day all-cause recidivism (LR test statistic $=0.31 ; \mathrm{p}=0.58$ ) and those with 30-day AHFS recidivism (LR test statistic $=0.88$; $\mathrm{p}=0.35$ ). The results were similar for both 5 -day all-cause and AHFS recidivism.

\section{Association of sST2 with Short-term Outcomes}

A majority of the outcomes we measured were noted on days 6-30 except for prolonged hospitalization and major cardiac events (Table 3). The data failed to show significant associations with sST2 and the 5-day outcomes in the unadjusted ( $\mathrm{p}=0.21$ and 0.14 for all-cause and AHFS restricted recidivism) or adjusted analyses (Table 4). Significant associations of SST2 with 30-day outcome were noted in the unadjusted analysis when recidivism was restricted to AHFS $(p=0.04)$. However, we failed to see a significant association between sST2 and all-cause recidivism $(\mathrm{p}=0.38)$. Median sST2 was higher in those who had a 30-day event where recidivism was restricted to $\operatorname{AHFS}(0.24 \mathrm{ng} / \mathrm{ml} ; \mathrm{IQR}=0.13$,

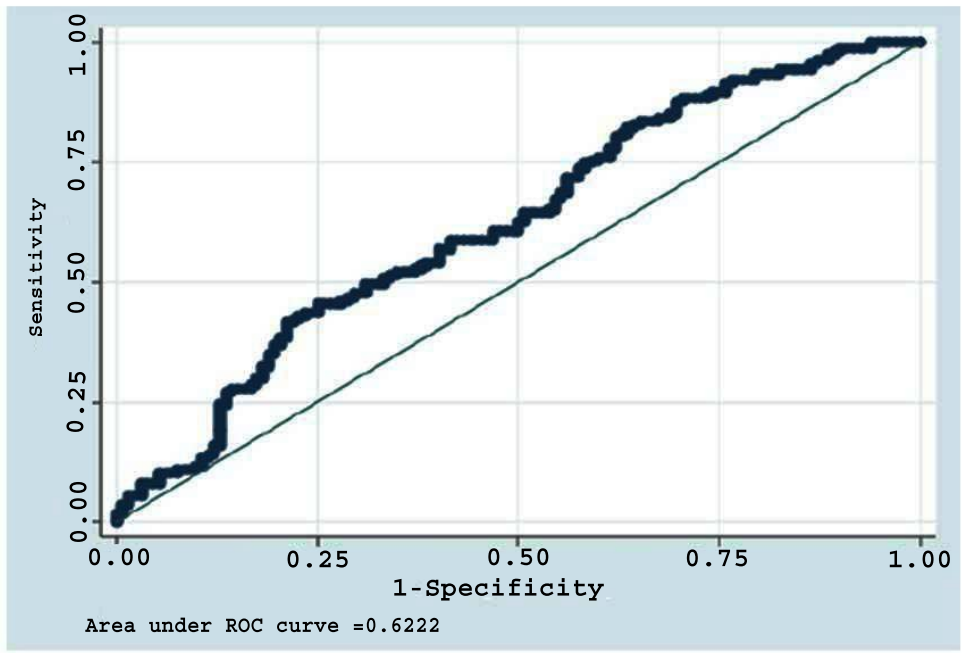

Fig. (1). Receiver operating characteristic curve for sST2 with the diagnostic outcome adjusting for prior aspirin and steroid use, age, gender, race and BMI. Area under the curve was 0.62 . 
Table 3. Frequency of 5 and 30-day Acute Heart Failure Syndromes (AHFS) Outcomes

\begin{tabular}{|c|c|c|}
\hline Adverse Outcomes & 5-day Adverse Outcomes n (\%) & 30-day Adverse Outcomes n (\%) \\
\hline \multicolumn{3}{|c|}{ Prolonged hospitalization ( $\geq 5$ or $\geq 30$ days) } \\
\hline No & $241(82)$ & $293(99)$ \\
\hline \multicolumn{3}{|c|}{ Unscheduled ED visit for HF } \\
\hline No & $289(98)$ & $275(93)$ \\
\hline \multicolumn{3}{|c|}{ Unscheduled ED visit not for $\mathrm{HF}$} \\
\hline No & $287(97)$ & $246(83)$ \\
\hline Yes & $8(3)$ & $49(17)$ \\
\hline \multicolumn{3}{|c|}{ Unscheduled hospital readmission for HF } \\
\hline No & $289(98)$ & $263(89)$ \\
\hline Yes & $2(1)$ & $29(10)$ \\
\hline \multicolumn{3}{|l|}{ Major cardiac event } \\
\hline No & $254(86)$ & $279(95)$ \\
\hline Yes & $41(14)$ & $16(5)$ \\
\hline \multicolumn{3}{|l|}{ Death (all cause) } \\
\hline No & $291(99)$ & $288(98)$ \\
\hline Yes & $4(1)$ & $7(2)$ \\
\hline
\end{tabular}

$0.46)$ than in those who did not have a 30-day event $(0.19$; $\mathrm{IQR}=0.08,0.32$ ); however, this difference may not be clinically significant. This significant association was not seen in the adjusted analysis (Table 4). Logistic regression models for heart failure status shows ORs for outpatient aspirin $(1.71 ; 95 \% \mathrm{CI}=1.05,2.77 ; \mathrm{p}=0.03)$ and steroid use $(0.36$; $95 \% \mathrm{CI}=0.18,0.73 ; \mathrm{p}=0.004$ ) (Table 4). The ORs for 5 - and 30-day HF only and all cause recidivism outcomes adjusted to the same covariates were not statistically significant.

\section{DISCUSSION}

We found that sST2 concentrations were increased in patients with AHFS compared to those without AHFS. Although the unadjusted analyses we conducted indicated that sST2 was significantly associated with the diagnostic and 30-day outcome with recidivism restricted to AHFS, this result did not hold true when we adjusted for outpatient use of aspirin and steroids, age, race, gender, and BMI. Further, sST2 does not appear to add independent diagnostic information to that provided by BNP.

Previous studies [23] noted sST2 concentrations were higher in patients with dyspnea attributable to AHFS than in patients with dyspnea attributable to other causes. They reported an AUC that was similar to our diagnostic AUC: 0.67 $(95 \% \mathrm{CI}=0.60,0.74)$ versus $0.62(95 \% \mathrm{CI}=0.56,0.69)$, respectively, for our current study.

In yet another study, the median concentrations of sST2 were reported to be significantly higher among ED patients with AHFS compared to those without ( 0.50 versus 0.15 $\mathrm{ng} / \mathrm{ml} ; \mathrm{p}<0.001)$, and in those who died compared to those who survived $(1.08$ versus $0.18 \mathrm{ng} / \mathrm{ml}, \mathrm{p}<0.001)$; there was also a strong relationship between $\mathrm{SST} 2$ concentrations and 1 year mortality (HR 5.6, $\mathrm{p}<0.001)[24,25]$. We failed to find any association between $\mathrm{sST} 2$ and prognosis in the adjusted analyses. This could be due to the fact that we looked at the 5- and 30-day outcomes, whereas Mueller et al., [24] evaluated the 1 year predictive capability of sST2 in patients with AHFS.

Another study [26] reported that serial sampling of sST2 concentrations had 90-day prognostic utility in patients admitted with AHFS. Plasma samples were collected at 6 time points between admission and discharge. Patients whose SST2 levels failed to decrease by $15.5 \%$ in this time period had a $33 \%$ chance of mortality. While this study has methodological differences from ours, it is noteworthy that serial sampling was suggested to have 90-day prognostic utility. These studies suggest the predictive ability of sST2 may be more useful long-term rather than short-term.

Natriuretic peptide testing is widely used in clinical practice and has been incorporated into cardiovascular guidelines for the diagnosis of AHFS [23, 27, 28]. Januzzi et al., [25] reported amino-terminal pro-brain natriuretic peptide (NTproBNP) was superior to sST2 for diagnosis of AHFS. They further noted that among dyspneic patients with AHFS, sST2 concentrations alone and in conjunction with NT-proBNP were predictive of mortality at 1-year. Our assessment sug- 
Table 4. Results from Logistic Regressions Models

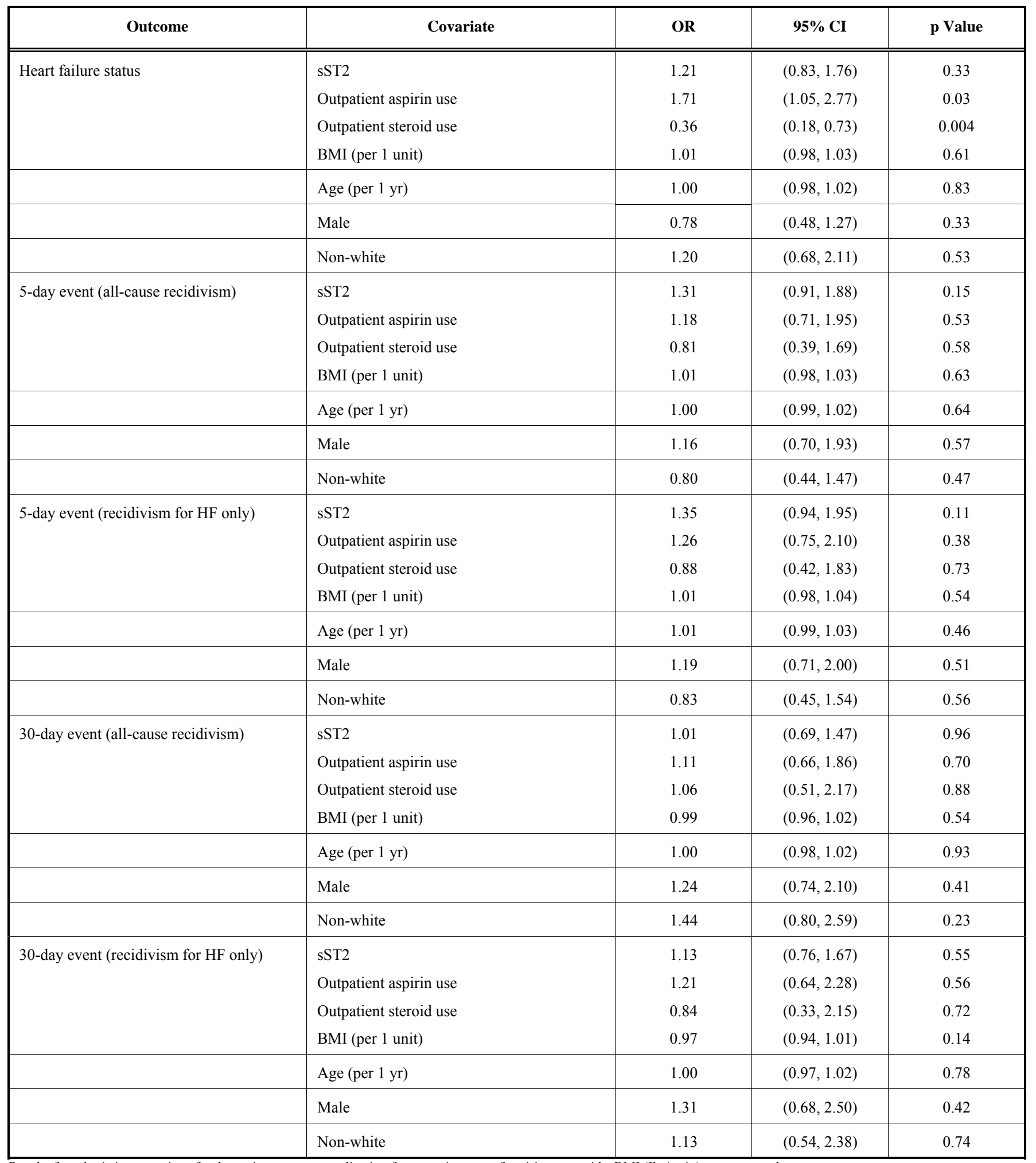

Results from logistic regressions for the various outcomes adjusting for outpatient use of aspirin or steroids, BMI (Ibs/sq.in), age, sex, and race OR, odds ratio; $\mathrm{CI}$, confidence interval; BMI, body mass index; $\mathrm{HF}$, heart failure.

gests sST2 did not improve BNP diagnostic or prognostic value at all BNP levels. Even in the gray zone area (BNP $100-500 \mathrm{pg} / \mathrm{ml}$ ), where the utility of BNP is debatable, sST2 failed to aid BNP in explaining the variability in the outcomes. 
Table 5. Likelihood Ratio Tests Assessing Additive Value of sST2 to BNP

\begin{tabular}{|l|c|c|c|c|c|}
\hline \multicolumn{1}{|c|}{ Outcomes } & & \multicolumn{2}{c|}{ Full data } & \multicolumn{2}{c|}{ BNP 100-500 pg/ml } \\
\hline \hline HF present / primary & Model & LR & p Value & LR & p Value \\
\hline 30-day (all-cause recidivism) & Main effects & 0.15 & 0.7 & 0.1 \\
\hline 30-day (HF recidivism only) & Main effects & 0.03 & 0.87 & 0.31 & 0.58 \\
\hline 5-day (all-cause recidivism) & Main effects & 0.18 & 0.68 & 0.88 & 0.35 \\
\hline 5-day (HF recidivism only) & Main effects & 2.01 & 0.16 & 1.89 & 0.17 \\
\hline
\end{tabular}

Results from likelihood ratio tests assessing whether sST2 (in the main effects model) or in the interaction of sST2 and BNP (in the model with the interaction) adds information to the logistic regression model in the full data and for subjects whose BNP fell between 100 and $500 \mathrm{pg} / \mathrm{ml}$.

BNP, B-natriuretic peptide; LR, likelihood ratio; HF, heart failure.

\section{CONCLUSION}

Soluble ST2 concentrations failed to show strong associations with diagnostic and prognostic outcomes in a cohort of ED patients with signs and symptoms of AHFS. Future studies looking at short-term outcomes may want to focus on the diagnostic and prognostic value of sST2 and its relationship with AHFS in a larger cohort of patients.

\section{CONFLICT OF INTEREST}

None declared.

\section{ACKNOWLEDGEMENTS}

The authors acknowledge the support of REDCap, Vanderbilt University (1UL1RR024975 from NCRR/NIH).

\section{REFERENCES}

[1] Flaherty JD, Bax JJ, De Luca L, et al. Acute heart failure syndromes in patients with coronary artery disease early assessment and treatment. J Am Coll Cardiol 2009; 53(3): 254-63.

[2] Gheorghiade M, Zannad F, Sopko G, et al. Acute heart failure syndromes: current state and framework for future research. Circulation 2005; 112(25): 3958-68.

[3] Braunwald E. Biomarkers in heart failure. N Engl J Med 2008; 358(20): 2148-59.

[4] Lee DS, Vasan RS. Novel markers for heart failure diagnosis and prognosis. Curr Opin Cardiol 2005; 20(3): 201-10.

[5] Kieser A, Goodnight J, Kolch W, Mischak H, Mushinski JF. Identification of the primary growth response gene, ST2/T1, as a gene whose expression is differentially regulated by different protein kinase C isozymes. FEBS Lett 1995; 372(2-3): 189-93.

[6] Weinberg EO, Shimpo M, Hurwitz S, Tominaga S, Rouleau JL, Lee RT. Identification of serum soluble ST2 receptor as a novel heart failure biomarker. Circulation 2003; 107(5): 721-6.

[7] Yanagisawa K, Tsukamoto T, Takagi T, Tominaga S. Murine ST2 gene is a member of the primary response gene family induced by growth factors. FEBS Lett 1992; 302(1): 51-3.

[8] Kumar S, Minnich MD, Young PR. ST2/T1 protein functionally binds to two secreted proteins from Balb/c $3 \mathrm{~T} 3$ and human umbilical vein endothelial cells but does not bind interleukin 1. J Biol Chem 1995; 270(46): 27905-13.

[9] Kuroiwa K, Li H, Tago K, Iwahana $\mathrm{H}$, et al. Construction of ELISA system to quantify human ST2 protein in sera of patients. Hybridoma 2000; 19(2): 151-9.

[10] Pascual-Figal DA, Ordonez-Llanos J, Tornel PL, et al. Soluble ST2 for predicting sudden cardiac death in patients with chronic heart failure and left ventricular systolic dysfunction. J Am Coll Cardiol 2009; 54(23): 2174-9.

[11] Mulrow CD, Lucey CR, Farnett LE. Discriminating causes of dyspnea through clinical examination. J Gen Intern Med 1993; 8(7): 383-92.

[12] Schmitt BP, Kushner MS, Wiener SL. The diagnostic usefulness of the history of the patient with dyspnea. J Gen Intern Med 1986; 1(6): 386-93

[13] Lindenfeld J, Albert NM, Boehmer JP, et al. HFSA 2010 Comprehensive Heart Failure Practice Guideline. J Card Fail 2010; 16(6): e1-194.

[14] Grantham JA, Borgeson DD, Burnett JC Jr. BNP pathophysiological and potential therapeutic roles in acute congestive heart failure. Am J Physiol 1997; 272(4 Pt 2): R107783 .

[15] Maisel AS, Krishnaswamy P, Nowak RM, et al. Rapid measurement of B-type natriuretic peptide in the emergency diagnosis of heart failure. N Engl J Med 2002; 347(3): 161-7.

[16] Maisel AS, Peacock WF, Shah KS, et al. Acoustic cardiography S3 detection use in problematic subgroups and B-type natriuretic peptide "gray zone": secondary results from the Heart failure and Audicor technology for Rapid Diagnosis and Initial Treatment Multinational Investigation. Am J Emerg Med 2011; 29(8): 924-31.

[17] Harrell FE Jr. Design: Design Package. 2009: Available from: http: //CRAN.R-project.org/package=Design .

[18] Zeileis A, Hothorn T. Diagnostic checking in regression relationships. R News. 2002; 2(3): 7-10.

[19] R Development Core Team. R: A language and environment for statistical computing. R Foundation for Statistical Computing. Vienna, Austria 2010; Available from: http: //www.R-project.org/.

[20] Harrell FE Jr. Hmisc: Harrell Miscellaneous. 2010; Available from: http: //CRAN.R-project.org/package $=$ Hmisc

[21] Hamilton LC, Stata Corporation. Stata release 11. College Station (Tex.): Stata Press; 2009.

[22] DeLong ER, DeLong DM, Clarke-Pearson DL. Comparing the areas under two or more correlated receiver operating characteristic curves: A nonparametric approach. Biometrics 1988; 44(3): 83745 .

[23] Dieplinger B, Gegenhuber A, Haltmayer M, Mueller T. Evaluation of novel biomarkers for the diagnosis of acute destabilised heart failure in patients with shortness of breath. Heart 2009; 95(18): 1508-13.

[24] Mueller T, Dieplinger B, Gegenhuber A, Poelz W, Pacher R, Haltmayer M. Increased plasma concentrations of soluble ST2 are predictive for 1-year mortality in patients with acute destabilized heart failure. Clin Chem 2008; 54(4): 752-6.

[25] Januzzi JL Jr., Peacock WF, Maisel AS, et al. Measurement of the interleukin family member ST2 in patients with acute dyspnea: results from the PRIDE (Pro-Brain Natriuretic Peptide 
Investigation of Dyspnea in the Emergency Department) study. J Am Coll Cardiol 2007; 50(7): 607-13.

[26] Boisot S, Beede J, Isakson S, et al. Serial sampling of ST2 predicts 90-day mortality following destabilized heart failure. J Card Fail 2008; 14(9): 732-8.
[27] Maisel A, Mehra MR. Understanding B-type natriuretic peptide and its role in diagnosing and monitoring congestive heart failure. Clin Cornerstone 2005; 7 (Suppl 1): S7-17.

[28] Maisel A, Mueller C, Adams K Jr, et al. State of the art: using natriuretic peptide levels in clinical practice. Eur J Heart Fail 2008; 10(9): 824-39.

Received: January 2, 2012

(C) Henry-Okafor et al.; Licensee Bentham Open.

This is an open access article licensed under the terms of the Creative Commons Attribution Non-Commercial License (http://creativecommons.org/licenses/by-nc/3.0/) which permits unrestricted, non-commercial use, distribution and reproduction in any medium, provided the work is properly cited. 